\title{
Three-dimensional joint inversion of gravity and magnetic anomalies based on density-magnetization relationship
}

\author{
Yosuke Teranishi, Hitoshi Mikada, Tada-nori Goto and Junichi Takekawa \\ ${ }^{1}$ Dept. of Civil and Earth Res. Eng., Kyoto University
}

\begin{abstract}
We present a three-dimensiolan (3D) joint inversion method to estimate two physical parameters, density and magnetization of subsurface materials using surface potential measurements (gravity and magnetic anomalies). In the method, we assume a linear relationship between the density and magnetization to relate gravity with magnetic data. In the approach, the subsurface structure is discretized to a set of rectangular prisms. For estimating the density and magnetization of each prism, we minimize the quadratic norm of the residuals between the observed and the forward-modeled potentials. Two regularization terms are used in our joint inversion. We also minimize the roughness and the similarity of the two physical parameters. The regularization parameters controlling the degree of roughness and similarity are determined in the L-curve criterion scheme. We apply our method to a numerical model which represents submarine massive sulphides (SMS). The joint inversion results, which have the advantages of both gravity and magnetic inversion, show better accuracy and resolution than the individual inversion results.
\end{abstract}

\section{Introduction}

The gravity and magnetic surveys have been widely used over the years, especially for metallic mineral deposits. These field intensity data can be acquired more quickly and simply than in the other geophysical and geological surveys. Therefore, these potential field surveys are often used at the preliminary stage of metallic mineral deposits. The inversion of the potential field data, however, has been known as a non-uniqueness problem expressed in the Green's equivalent layer theory. Because of this problem, gravity and magnetic data have no inherent resolution in depth. As generic surveys, the measurements of potential fields are powerful schemes before any specific surveys. But, the utilization of potential field data requires subsurface model or some other data acquired by the other means for modeled or joint inversion.

Actually, a joint inversion using geostatistic method of gravity and magnetic data sets is known useful for lithology discrimination and classification $^{1)}$. Also, joint inversion using cross-gradient function of two disparate geophysical data sets increases accuracy and resolution of three-dimensional geophysical models $^{2}$. Several studies have been done proposing various methods to associate gravity data with magnetic data, how to define their object functions and regularization parameters. The past examples indicate that we could develop a method to utilize both gravity and magnetic data using joint inversion algorithms.

In this paper, we develop a $3 \mathrm{D}$ joint inversion technique assuming a relationship between subsurface density and magnetization. The regularization parameters could be determined using the L-curve criterion scheme. We test our inversion method on synthetic numerical experiments, and compare the results from our joint inversion with the other inversion that deals with potential field data independently. For numerical tests, we focus on submarine massive sulfide (SMS) as an exploration target, since SMS are thought to be one of the new mineral resources. Although several surveys have been conducted for SMS using gravity or magnetic measurements ${ }^{3), 4)}$, there is no report that succeeded to estimate the amount of resources accurately enough. We apply our joint inversion method to demonstrate the accuracy of our method in the estimation of subsurface density and magnetic anomalies for estimating the amount of resources quantitatively.

\section{Method}

\section{(1) Formulation of 3D inversion}

Gravity and magnetic anomaly observed above the surface with $\mathrm{N}$ of observations (gravity anomaly vector $\mathbf{d}_{\mathbf{g}}$, magnetic anomaly vector $\mathbf{d}_{\mathbf{m}}$ ) are related to density $\boldsymbol{\rho}$ and magnetization $\mathbf{m}$ of $\mathrm{M}$ 
underground prisms linearly:

$$
\begin{gathered}
\mathbf{d}_{\mathrm{g}}=\mathbf{A}_{\mathrm{g}} \boldsymbol{\rho} \\
\mathbf{d}_{\mathbf{m}}=\mathbf{A}_{\mathbf{m}} \mathbf{m}
\end{gathered}
$$

The matrix ( $\left.\mathbf{A}_{\mathbf{g}}, \mathbf{A}_{\mathbf{m}}\right)$ have elements $\left(\mathbf{A}_{\mathbf{g} i j}, \mathbf{A}_{\mathbf{m} i j}\right)$, which are the contributions of unit density and magnetization in the $j$ th prism to the $i$ th data. $\left(\mathbf{A}_{\mathbf{g} i j}\right.$, $\left.\mathbf{A}_{\mathbf{m} i j}\right)$ consist of constants, so our inversion is linear algorism. The first step in setting up the inversion is to define a misfit function. Here we use the L-2 norm term $S_{g}$ (gravity data misfit) and $S_{m}$ (magnetic data misfit).

$$
\begin{aligned}
& S_{g}=\left\|\mathbf{W}_{\mathbf{g}}\left(\mathbf{d}_{\mathbf{g}}-\mathbf{A}_{\mathbf{g}} \mathbf{\rho}\right)\right\|^{2} \\
& S_{m}=\left\|\mathbf{W}_{\mathbf{m}}\left(\mathbf{d}_{\mathbf{m}}-\mathbf{A}_{\mathbf{m}} \mathbf{m}\right)\right\|^{2}
\end{aligned}
$$

$\left(\mathbf{W}_{\mathrm{g}}, \mathbf{W}_{\mathrm{m}}\right)$ are weighting matrices. If noise contaminating each data is uncorrelated and has a standard deviation of $\left(\sigma_{g i}, \sigma_{m i}\right)$, then the weighting matrices are usually defined as $\mathbf{W}_{\mathbf{g}}=\operatorname{diag}\left\{1 / \sigma_{g l}, \ldots\right.$, $\left.1 / \sigma_{g n}\right\}, \mathbf{W}_{\mathbf{m}}=\operatorname{diag}\left\{1 / \sigma_{m l}, \ldots, 1 / \sigma_{m n}\right\}$. The notation $\|\cdot\|$ in the above two equations is the L-2 norm measure. However, this method is often difficult to make an extremely accurate initial solution, and this method includes parameters which are poorly determined. As a result, the solution is unstable and ambiguous. Therefore, in the second step, we add a regularization term to our algorism to avoid unstableness and ambiguity, and that the effect of noise in the data is minimized. Furthermore, we add a smoothing Laplacian operator matrix $\mathbf{C}$ as a smoothness regularization term to our algorism and we obtain following objective functions.

$$
\begin{aligned}
& U_{g}=\left\|\mathbf{W}_{\mathbf{g}}\left(\mathbf{d}_{\mathbf{g}}-\mathbf{A}_{\mathbf{g}} \mathbf{\rho}\right)\right\|^{2}+\alpha_{g}{ }^{2}\|\mathbf{C} \boldsymbol{\rho}\|^{2} \\
& U_{m}=\left\|\mathbf{W}_{\mathbf{m}}\left(\mathbf{d}_{\mathbf{m}}-\mathbf{A}_{\mathbf{m}} \mathbf{m}\right)\right\|^{2}+\alpha_{m}{ }^{2}\|\mathbf{C} \mathbf{m}\|^{2}
\end{aligned}
$$

Smoothness regularization parameters $\alpha_{\mathrm{g}}$ and $\alpha_{\mathrm{m}}$ control a degree of spatial smoothing in inversion. Since inversion results are affected by the smoothness regularization parameters $\alpha_{\mathrm{g}}$ and $\alpha_{\mathrm{m}}$, it is very important to determine $\alpha_{g}$ and $\alpha_{m}$ adequately.

\section{(2) Relationship between density with magnetization}

Here we state association strategy between the density and magnetization. According to Bosch $(1999)^{1)}$, they associated the density with magnetization in each different rock using the
Gaussian models. On the basis of Bosch (1999) ${ }^{1)}$, we consider a simple linear relationship between two parameters. In the former studies ${ }^{3), 4)}$, the SMS generally possesses higher density and lower magnetization than the surrounding volcanic rocks. For simplification and idealization, we suppose following linear relationship between anomalous density $\boldsymbol{\rho}$ and anomalous magnetization $\mathbf{m}$ in SMS.

$$
\mathbf{m}=a \rho
$$

$a$ is a arbitrary number. In the surrounding area, the anomaly of $\boldsymbol{\rho}$ and $\mathbf{m}$ are assumed to be zero as a background values.

\section{(3) Formulation of the 3D joint inversion}

To formulate our joint inversion method, we incorporate equation (5) with (6) through equation (7) as a similarity regularization term which combines two model parameters to formulate a joint inversion algorithm and we obtain the following equation.

$$
\begin{aligned}
U & =\left\|\mathbf{W}_{\mathbf{g}}\left(\mathbf{d}_{\mathbf{g}}-\mathbf{A}_{\mathbf{g}} \boldsymbol{\rho}\right)\right\|^{2}+\left\|\mathbf{W}_{\mathbf{m}}\left(\mathbf{d}_{\mathbf{m}}-\mathbf{A}_{\mathbf{m}} \mathbf{m}\right)\right\|^{2} \\
& +\alpha_{g}{ }^{2}\|\mathbf{C} \boldsymbol{\rho}\|^{2}+\alpha_{m}{ }^{2}\|\mathbf{C} \mathbf{m}\|^{2}+\lambda^{2}\|\mathbf{F}(\boldsymbol{\rho}, \mathbf{m})\|^{2}
\end{aligned}
$$

where, $\mathbf{F}(\boldsymbol{\rho}, \mathbf{m})=\mathbf{m}-a \boldsymbol{\rho}$

The smoothness regularization parameter $\alpha_{\mathrm{g}}$ and $\alpha_{m}$ controls the weight of smoothness constraint against the data misfit and the model similarity. Also the similarity regularization parameter $\lambda$ controls the weight of similarity constraint against the data misfit and the model smoothness. The parameter $\beta$ controls the weighting ratio of gravity and magnetic in data misfit. In the joint inversion, two model parameters, density and magnetization, are following equation (7). So we can obtain following relationship between $\alpha_{\mathrm{g}}$ and $\alpha_{\mathrm{m}}$;

$$
\alpha_{\mathrm{m}=} a \alpha_{\mathrm{g}}
$$

We have to consider linear relationship value $a$ as well as $\beta, \alpha_{\mathrm{m}}$ and $\lambda$. In these parameters, regarding to $\beta$ and $\lambda$, we pre-select these values as prior information and regarding to $\alpha_{\mathrm{m}}$ and linear relationship value $a$, we use an L-curve criterion scheme to determine $\alpha_{m}$ and linear relationship value $a$ in equations (8).

\section{(4) L-curve criterion scheme}

When model roughness is plotted as a function of rms with smoothing regularization parameter, the plots show the curve like L-shaped in each 
relationship.

And we select the point minimizing distance from the origin as the optimum point. Because the $\alpha$ and a of this optimum point have nice balance between rms and model roughness.

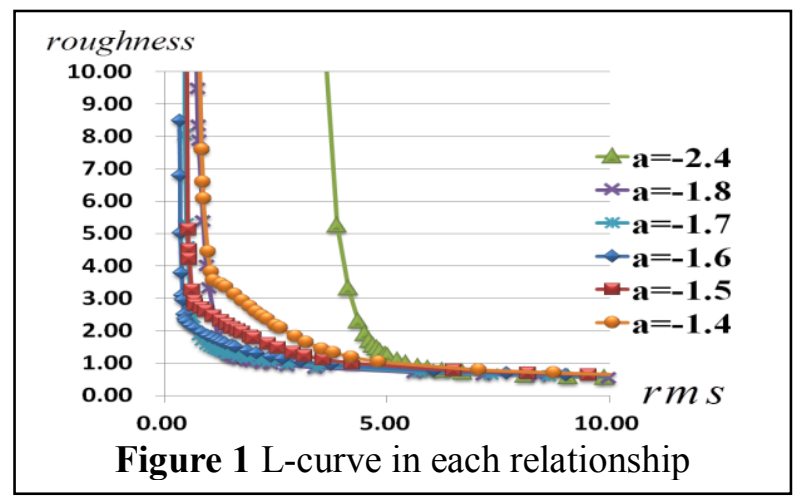

\section{Synthetic test model}

In order to test our joint inversion algorithm, we consider the SMS model shown in Figure 2. The model consists of $15 \times 15 \times 12$ prisms in the analysis model that has $300 \mathrm{~m}$ in both horizontal directions and $96 \mathrm{~m}$ in depth. Based on Evans (1996) ${ }^{3)}$; Tivey et al. $(2002)^{4)}$, we supposed the difference in density between SMS and surrounding volcanic rock is $2.5 \mathrm{~g} / \mathrm{cm}^{3}$ and that of magnetization is $-4.0 \mathrm{~A} / \mathrm{m}$. We calculate anomalous data on the surface over a $17 \times 17$ grid with $20 \mathrm{~m}$ spacing in both directions. $0.01 \mathrm{mGal}$ and $5 \mathrm{nT}$ Gaussian noise is added to the synthetic data. The observation level is assumed to be $1 \mathrm{~m}$ above the seabed, and the background magnetic field has constant inclination and declinations; $I=45^{\circ}, D=0^{\circ}$. The same inclination and declination are assigned for the magnetization vectors of rocks.

\section{Synthetic examples}

Figure 3 shows the results of four cross-sections of density and magnetization structure at $x=150 \mathrm{~m}$ obtained from after separate inversion using gravity data, separate inversion using magnetic data and joint inversion. Furthermore, we estimated respective regularization smoothness parameters $\alpha_{\mathrm{g}}$, $\alpha_{\mathrm{m}}$ and linear relationship value $a$ using the L-curve criterion. Now, the regularization smoothness parameter $\lambda$ is fixed to 20 .

In the both separated gravity and magnetic inversion results, the estimated models do not clearly indicate the stepwise shapes of assumed anomalous blocks. On the other hand, those by our joint inversion show good agreement with the true model, especially around boundary of stepwise structure.

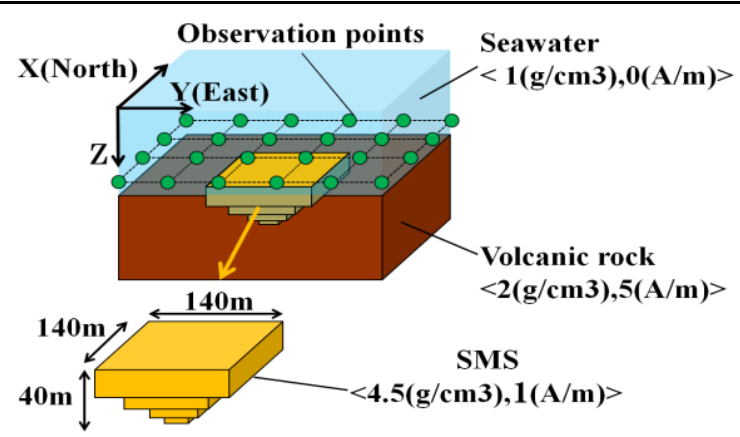

Figure 2 The stepwise SMS models

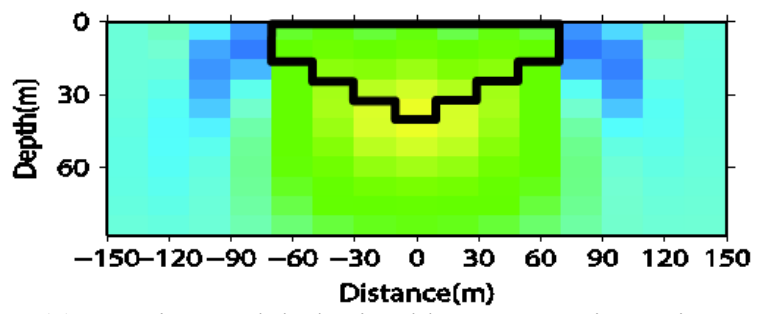

(a) Density model obtained by separate inversion

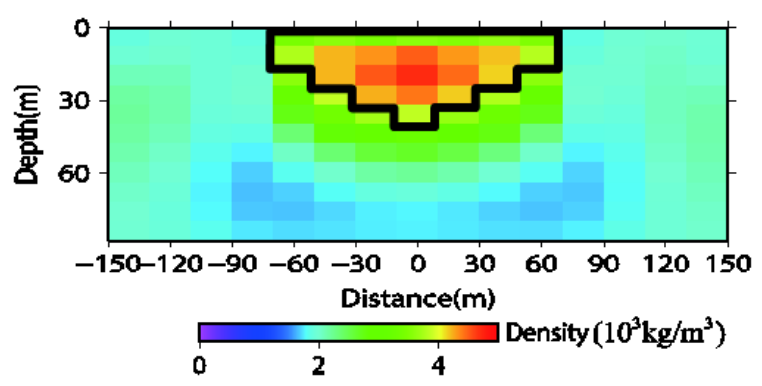

(b) Density model obtained by joint inversion

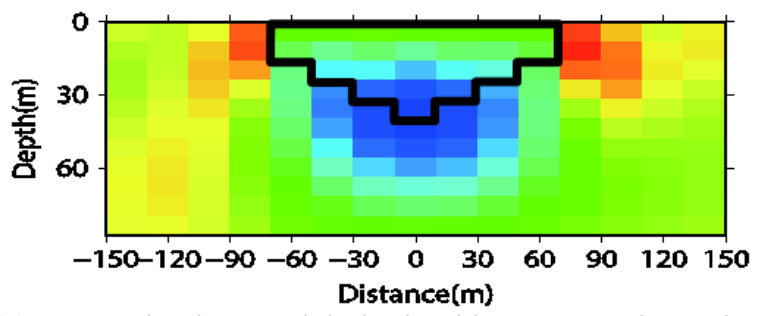

(c) Magnetization model obtained by separate inversion

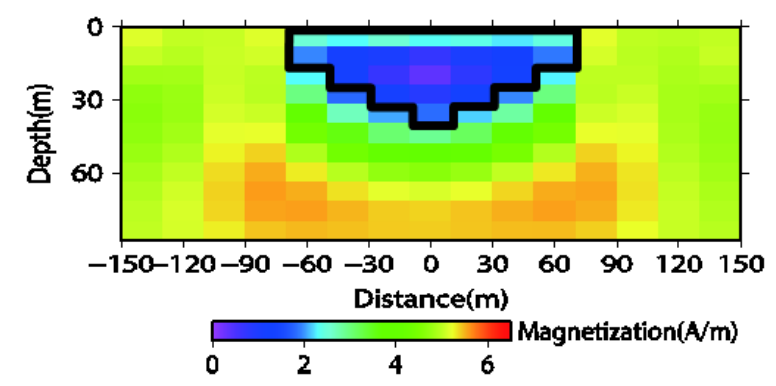

(d) Magnetization model obtained by joint inversion

Figure 3 (a) Density and (c) magnetization models obtained by separate inversion; (b) density and (d) magnetization models obtained by joint inversion. The vertical axis indicates depth below the seabed and the cross axis indicates horizontal distance. The true position of the model is outlined in each cross-section. 


\section{Discussion}

In our results mentioned above, joint inversion using gravity and magnetic data improved the resolution in comparison with the individual ones. In order to argue the cause of the improvement due to the joint inversion, we calculate a sensitivity how a unit quantity variation of density and/or magnetization in each prism affects the anomalous data regarding each inversion method. We compute a sum of each sensitivity matrix (Jacobian) in direction of suffix $i$ as shown below.

$$
\text { Sensitivity }=\sum_{i=1}^{N} A_{i j}
$$

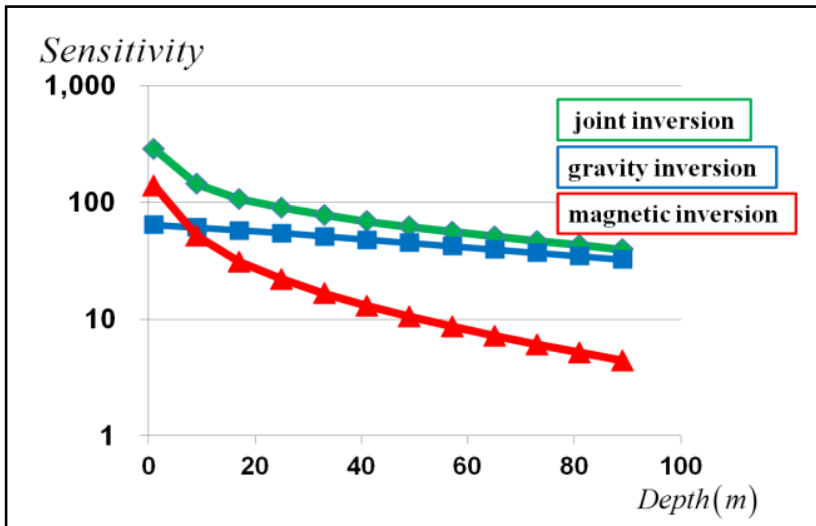

Figure 4: Logarithm of sensitivity of each inversion. The vertical axis indicates values of logarithm of the sensitivity and the cross axis indicates depth below the seabed. Blue, red and green curves show sensitivity of gravity, magnetic and joint inversion respectively.

Figure 4 shows the logarithmic sensitivity of each inversion method. The sensitivity of magnetic inversion is higher than gravity inversion near the surface but rapidly decreases with depth. On the other hand, the sensitivity of gravity inversion decreases gradually compared to that of magnetic inversion. These curves are consistent with the fact that gravity and magnetic fields decay with the square and cubic of distance, respectively. Joint inversion has the advantages of both methods showing gradual decrease in sensitivity with depth and highest sensitivity at zero depth. Because of this, joint inversion has narrowed down the space of possible solutions deduced, by the inversions of each single physical parameter.

\section{Conclusion}

We calculated the noise-contaminated synthetic data generated by the SMS model. We have developed an algorithm to jointly invert two geophysical data sets associated a constraint with linear relationship between the density and magnetization. In the individual inversion results, the resolution and accuracy of inverted results indicated the limitation in boundary value problems. On the other hand, in our joint inversion results, the inverted results show better resolution and accuracy. The combination of two physical parameters having different distance dependence might have relaxed the limitations of boundary value problems.

1) Bosch, M., 1999, Lithologic tomography: From plural geophysical data to lithology estimation: Journal of Geophysical Research, 104, 749-766.

2) Fregoso, E., and L. A. Gallardo, 2009, Cross-gradients joint 3D inversion with applications to gravity and magnetic data: Geophysics, 74, no.4, L31-L42.

3) Evans, R. L., 1996, A seafloor gravity profile across the TAG hydrothermal mound: GEOPHYSICAL RESEARCH LETTERS: 23, 3447-3450

4) Tivey, M. A., and H. P. Johnson, 2011, Crustal magnetization reveals subsurface structure of Juan de Fuca Ridge hydrothermal vent fields: Geological Society of America, 30, 979-982 